\title{
La lacune de Stafne : à propos d'un cas
}

\section{RÉSUMÉ}

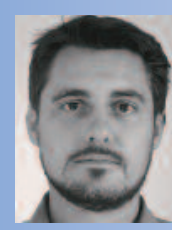

Rémi CURIEN

Assistant hospitalier universitaire, Faculté d'Odontologie de Nancy, Département de Chirurgie Buccale, Service d'Odontologie,

CHU de Nancy.

96, avenue du Maréchal de Lattre de Tassigny, BP 50208

54000 Nancy.

Julien BALLY

Assistant hospitalier universitaire, Faculté d'Odontologie de Nancy, Département de Chirurgie Buccale, Service d'Odontologie,

CHU de Nancy.

\section{Alexandra SOURDOT}

Assistant hospitalier universitaire, Faculté d'Odontologie de Nancy, Département de Chirurgie Buccale, Service d'Odontologie,

CHU de Nancy.

\section{Pierre BRAVETTI}

Maître de conférence

Praticien hospitalier

à la faculté d'Odontologie de Nancy,

Doyen de la faculté d'Odontologie,

Responsable du département

de Chirurgie et Pathologie Buccale,

Service d'Odontologie,

CHU de Nancy.
La lacune de Stafne est une cavité développée aux dépends de la face linguale de la mandibule, vraisemblablement par pression de la glande sous-mandibulaire. Elle est asymptomatique et ne nécessite aucun traitement. Son diagnostic est essentiellement radiologique et est indispensable afin d'éviter un geste chirurgical inutile et risqué. 


\section{Introduction}

$>$

Stafne, en 1942 [1], rapporte pour la première fois 35 cas de lacunes osseuses asymptomatiques et non évolutives situées dans la région postérieure de la mandibule. Depuis, de multiples appellations ont été affectées à cette lésion (tab. I). La classification de I'OMS reconnaît les termes de " latent bone cyst of jaw " et de "Stafne's Cyst » [2] dans l'intitulé Developmental disorders of jaws.

Dans les pays francophones, le terme de lacune de Stafne est le plus communément admis.

La lacune a radiologiquement un aspect ovoïde à contours bien définis, d'allure kystique. Elle est délimitée par un liseré d'ostéocondensation qui serait, d'après certains auteurs [3], plus fin et plus dense que celui des kystes odontogènes.

Sa situation peut varier de l'angle mandibulaire à la première molaire définitive, mais elle se situe toujours sous le canal mandibulaire [1, $4,5,6,7,8]$. Un point important pour l'affirmation du diagnostic est la stabilité au cours du temps de cette image radiologique [9]. Des variants plus rares ont été décrits dans la région mandibulaire antérieure et dans la branche mandibulaire $[8,10]$.

Son incidence est de 0,1 à $0,48 \%$ en cas de découverte radiologique mais serait en réalité plus proche des $6 \%$ dans la population générale $[4-6,8]$.

Sa prédilection pour le sexe masculin et sa plus grande fréquence de diagnostics entre 40 et 60 ans sont clairement établis $[4,5,8,11]$.

Diverses hypothèses étiopathogéniques ont été proposées. La majorité des auteurs considère actuellement que la lacune résulte de la pression exercée par la glande sous-mandibulaire (ou sublinguale dans le variant antérieur, parotide dans le variant ramique) sur la corticale linguale de la mandibule $[4-6,8,12]$. Cette théorie est étayée par des preuves sialographiques et chirurgicales.

D'autres facteurs ont été évoqués, tels qu'un défaut d'ossification dans la zone primitivement occupée par le cartilage de Meckel ou la pression de l'artère faciale $[1,8,11,13]$.

Nous présentons ici un cas de lacune de Stafne diagnostiquée fortuitement au cours d'un examen radiographique.

\begin{tabular}{l} 
Dénominations dans la littérature [4, 9]. \\
\begin{tabular}{|l|}
\hline Embryonic mandibular defect \\
\hline Latent osseous cyst \\
\hline Static osseous csyt / defect / cavity \\
\hline Aberrant salivary gland defect \\
\hline Mandibular inclusion of salivary glands \\
\hline Osseous idiopathic cavity \\
\hline Stafne's cyst / Stafne's bone cyst \\
\hline Mandibular lingual depression / concavity \\
\hline Developmental mandibular osseous defect \\
\hline $\begin{array}{l}\text { Developmental mandibular lingual glandular depression } \\
\text { / defect (posterior variant) }\end{array}$ \\
\hline
\end{tabular} \\
\hline
\end{tabular}




\section{Description du cas}

Un patient de 54 ans, sans antécédent particulier, est adressé en consultation par son chirurgien dentiste suite à la découverte fortuite sur un orthopantomogramme (fig. 1) d'une radioclarté dans le corps mandibulaire gauche. Le patient ne rapporte aucun symptôme.

L'examen clinique ne retrouve aucune voussure ou douleur à la palpation du corps mandibulaire.

L'orthopantomogramme met en évidence une lésion radioclaire ovalaire allongée d'avant en arrière, de 2 centimètre de grand axe, entourée d'un net liseré d'ostéocondensation. L'image se situe entre le bord basal et la corticale supé- rieure du canal mandibulaire, et n'entretient aucun rapport avec les dents.

Tous les signes collectés étant en faveur d'une lacune de Stafne, un examen tomodensitométrique est prescrit au patient afin de confirmer la continuité de la lésion avec le plancher buccal, signe diagnostique essentiel de cette pathologie. L'image fournie par le Dentascan (fig. 2 et 3) confirme effectivement cet aspect et le complète en faisant état de sa nature adipeuse.

L'attitude thérapeutique adoptée est l'abstention assortie d'une surveillance à 1 an puis 5 ans afin de s'assurer de l'absence d'évolution de la lésion.
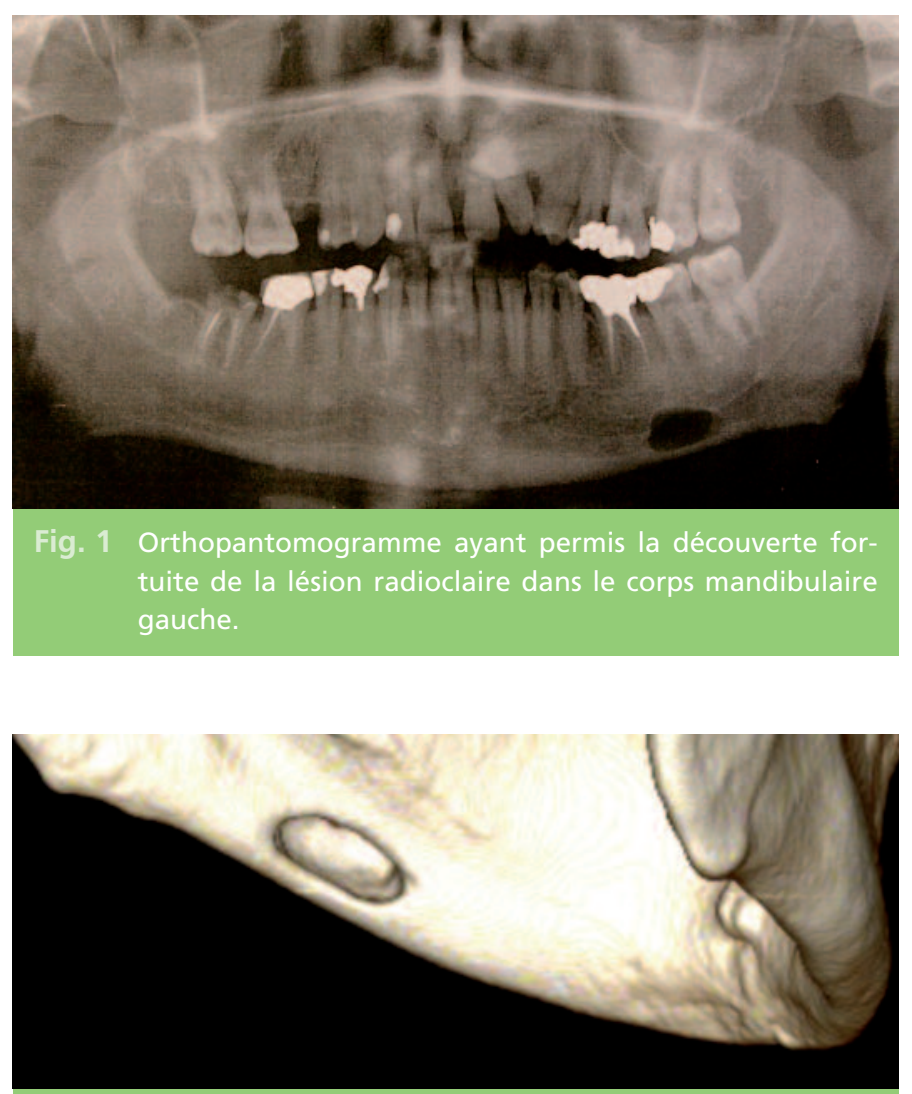

Fig. 3 Reconstitution tomodensitomètrique en trois dimensions du corps mandibulaire gauche, en vue médio-postérieure.

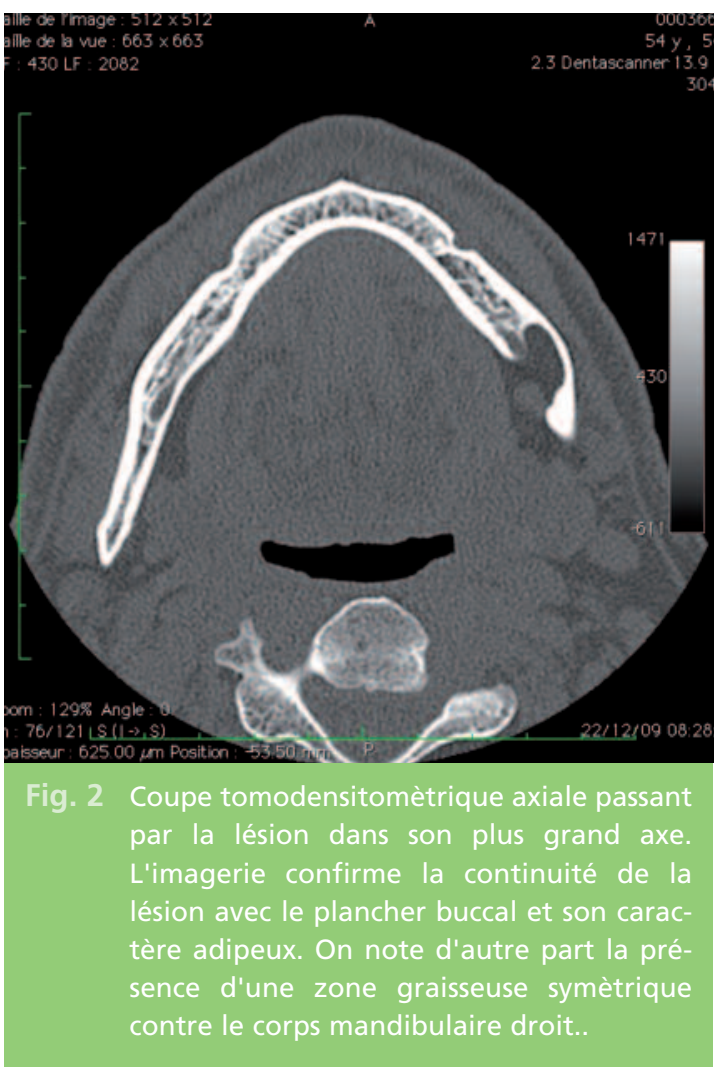




\section{Discussion}

La lacune de Stafne étant totalement asymptomatique, son diagnostic est habituellement fortuit et radiologique. C'est l'orthopantomogramme qui, le plus souvent, révèlera une radioclarté très évocatrice $[1,4,7]$. L'aspect radiologique typique, doublé du caractère asymptomatique, permet d'évoquer le diagnostic mais ne suffit pas à l'affirmer. Des examens d'imagerie complémentaire doivent donc être pratiqués. Le choix doit se faire entre la tomodensitométrie, la sialographie et I'IRM.

La tomodensitométrie est actuellement considérée comme l'examen complémentaire de choix [4]. Des coupes axiales permettent d'observer la concavité osseuse linguale $[9,13,14]$ et d'en déterminer le contenu. En effet, si le contenu de la lacune de Stafne est le plus souvent salivaire, il peut s'agir plus rarement de structures fibreuses, lymphoïdes, musculaires, vasculaires ou adipeuses [15, 16]. Ariji et al. [13] ont établi une classification du contenu radiologique de la cavité : le type $\mathrm{F}($ fat) a une densité de type " graisse »; dans le type G (gland), la glande sous-mandibulaire émet un prolongement dans la cavité ; le type S (soft tissue) présente une densité de tissus mous pouvant correspondre à un ganglion lymphatique ou à du tissus conjonctif ou vasculaire.

Dans le cas présent, la tomodensitométrie a permis de visualiser un contenu adipeux.

La sialographie permet de confirmer et de visualiser le contenu glandulaire de la lacune [11] mais se révèle inadaptée en cas de contenu non glandulaire. La littérature rapporte en outre des faux négatifs [17].

Selon Segev, Puterman et Bodner [7], la radiologie conventionnelle et la tomodensitométrie permettent de déterminer uniquement la forme et les contours de la cavité mais ne peuvent en diagnostiquer avec certitude le contenu. L'IRM offre un meilleur contraste des tissus mous (même sans produit de contraste) et serait de ce point de vue plus performante [7]. Ses inconvénients majeurs sont son coût, sa difficulté d'accès ainsi que les artefacts produits par les matériaux de restauration dentaire. Elle pourrait être envisagée comme un examen de seconde intention, en cas d'échec diagnostique avec la tomodensitométrie.

Cependant, seul l'examen histologique serait à même de fournir le diagnostic de certitude.

Le bilan radiologique ne fournira qu'un diagnostic de probabilité. Si le diagnostic de lacune de Stafne est l'hypothèse la plus probable, l'abstention assortie d'une surveillance régulière est l'attitude thérapeutique la plus appropriée [4-6, 8]. Si le suivi révèle une évolution de la lésion, la chirurgie et un examen histopathologique du contenu de la lacune sont indiqués. Signalons pour mémoire le cas d'une supposée lacune de Stafne dont le contenu s'est révélé être... un adénome pléomorphe [18].

Pour conclure, le diagnostic positif d'une lacune de Stafne se base sur un faisceau d'arguments :

- aspect pseudo-kystique bien délimité ;

- indépendance vis-à-vis des dents ;

- situation sous le canal mandibulaire ;

- absence de signes cliniques tels que des douleurs ou une tuméfaction,

- affirmation de la continuité de son contenu avec une structure pelvibuccale ,

- enfin (et surtout), absence d'évolution à court, moyen et long terme.

La lacune de Stafne est une lésion importante à connaître par tout praticien car elle peut conduire à tort à un geste chirurgical inutile et de surcroît risqué. En raison de son aspect typique, elle est de diagnostic relativement aisé. 


\section{Bibliographie}

1. Stafne $E$

Bone cavities situated

near the angle

of the mandible.

J Am Dent Assoc

1942;29:1969-72.

2. World Health Organization. International statistical classification of diseases and related health problems.

$10^{\text {th }}$ edition, 2007.

3. Uemura S, Fujishita M, Fujishita $\mathrm{H}$.

Radiographic interpretation of so-called developmental defects of the mandible. Oral Surg Oral Med Oral Pathol 1976;41:120-8.

4. Gómez $\mathrm{CQ}$, Castellón EV, Aytés LB, Escoda CG. Stafne bone cavity: a retrospective study of 11 cases.

Med Oral Pathol Oral

Chir Buccal 2006;11:E277-80.

5. Grellner T, Frost $D$, Brannon R.

Lingual mandibular bone defect:

Report of three cases.

J Oral Maxillofac Surg 1990;48:288-96.

6. Slasky B, Bar-Ziv J.

Lingual mandibular bony defects:

CT in the buccolingual plane.

J Comput Assit Tomogr 1996;20:438-43.

7. Segev $Y$, Puterman $M$, Bodner L. Stafne bone cavity. Magnetic resonance imaging.
Med Oral Pathol Oral

Chir Buccal 2006;11:E345-7.

8. Philipsen $\mathrm{H}$, Takata $\mathrm{T}$, Reichart P, Sato S, Suei Y. Lingual and buccal mandibular bone depressiones:

A review based on $\mathbf{5 8 3}$ cases from a world-wide literature survey, including 69 new cases from Japan. Dentomaxillofac Radiol 2002;31:281-90

9. Junquera LM, Albertos JM, Ferreras J, Baladron J.

Stafne Bone cyst.

Revision of four cases, one of them

with bilateral affectation.

Ann Otolaryngol

Chir Cervicofac 1999;116:295-298.

10. De Courten $A$, Küffer $R$, Samson J, Lombardi T.

Anterior lingual mandibular salivary gland defect (Stafne defect) presenting as a residual cyst.

Oral Surg Oral Med Oral Pathol Oral Radiol Endod 2002;94(4):460-4.

11. Barker G.

Xeroradiography in relation to a Stafne bone cavity. $\mathrm{Br} J$ Oral Maxillofac Surg 1988; 26:32-5.

12. Tolman DE, Stafne EC. Developmental bone defects of the mandible. Oral Surg Oral Med Oral Pathol 1967;24:488-90.

13. Ariji E, Fujiwara N, Tabata O, Nakayama E, Kanda S, Shiratsuchi Y, Oka M.
Stafne's bone cavity.

Classification based on outline and content determined by computer tomography.

Oral Surg Oral Med

Oral Pathol 1993;76:375-80.

14. Marsot-Dupuch $K$, Katz $P$, Maulat I, Quillard J, TassartMet Doyon D. Imagerie des glandes salivaires.

Encycl Méd Chir (Paris : Elsevier), Radiodiagnostic - Appareil digestif, 33-020-A-10, 2003.

15. Gorab GN, Brahney C, Aria AA.

Unusual presentation of a Stafne bone cyst. Oral Surg Oral Med Oral Pathol,1986;61:213-20.

16. Brygo A, Leroy $X$, Maes JM, Ferri J.

Tumeurs et pseudotumeurs non odontogènes bénignes des maxillaires.

EMC (Paris : Elsevier), Stomatologie, 22-062-H-10, 2006, Médecine buccale, 28-550-M-10, 2008.

17. Oikarinen VJ, Wolf J, Julku M.

A sterosialographic study of developmental mandibular bone defects (Stafne's idiopathic bone cavities). Int J Oral Surg 1975;4:51-4.

18. Simpson $\mathrm{W}$.
A Stafne's mandibular defect containing a pleomorphic adenoma.
J Oral Surg 1965;23:553-6. 


\title{
SUMMARY
}

\section{Stafne's bone cavity:}

report of a case

Rémi CURIEN,

Julien BALLY,

Alexandra SOURDOT,

Pierre BRAVETTI

\section{Keywords \\ jaw diseases \\ developmental bone \\ disease \\ submandibular gland \\ diseases \\ CT scan}

Stafne's bone cyst is an osseous cavity on the lingual face of the mandible, probably caused by the pression of the submandibular gland. It is asymptomatic and doesn't require any treatment. Its diagnosis is mostly radiologic and is essential to avoid useless surgery.

\section{La mise en acte du traitement : \\ Les six dimensions de la relation thérapeutique}

\author{
M.G. Choukroun
}

Dans cet ouvrage, la relation praticien/patient est formalisée de telle sorte que le lecteur pourra appliquer sans difficulté les notions évoquées à sa clinique.

Cette relation se fonde sur la maîtrise de six dimensions : le biomédical, l'information, l'éducation, le transfert, le psycho-social et l'encadrement.

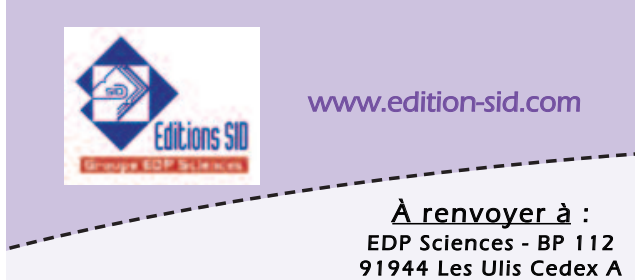

Nom / Prénom : Adresse

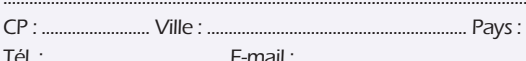

1944 Les Ulis Cedex A

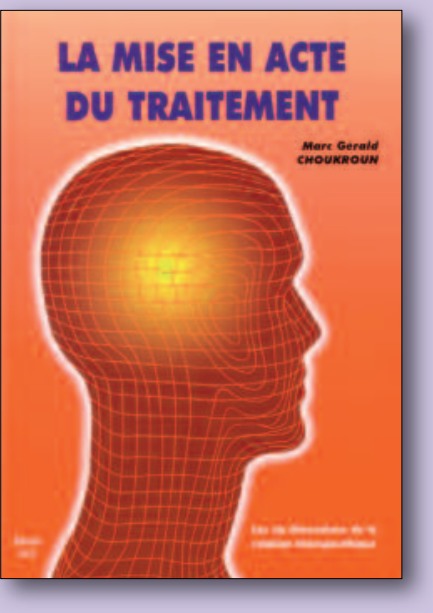

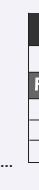
Lais de port acte du traitement France métropolitan \begin{tabular}{ll|l|l} 
DOM et Europe & $+7 \epsilon$ & $+9 \epsilon$ & $+11 \epsilon$ \\
\hline TOM et reste du monde & $+9 \epsilon$ & $+12 \epsilon$ & +15
\end{tabular} Paiement:

口par chèque à l'ordre d'EDP Sciences là joindre à la commande $\square$ par carte bancaire: $\quad \square$ Visa $\quad \square$ Eurocard $\square$ American Express

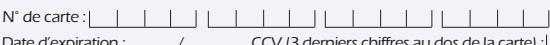


France et Union Européenne version papier + version électronique $204 €$

Étudiant* : France et Union Européenne

(* sur présentation d'une attestation) version papier + version électronique $124 €$ Reste du monde version papier + version électronique

Membre de I'AEOS

Prix au numéro

Frais d'envoi par avion

(*) Offre valable jusqu'au 31/10/2010
4 NUMÉROS(*)

TTC

version électronique seule

$179 €$

version électronique seule $109 €$ version électronique seule $149 €$ $234 €$ $183 €$

$67 €$ $49 €$
Frais de port au numéro $\quad 4 €$

$\square$ Je souhaite $\mathbf{m}$ 'abonner à la Revue AOS

$\square$ Je souhaite prolonger mon abonnement à la Revue AOS

\section{MODE DE PAIEMENT :}

$\square$ Paiement effectué par chèque à l'ordre de : EDP Sciences - REVUE AOS

17 avenue du Hoggar - P.A. de Courtabœuf

B.P. 112 - 91944 Les Ulis Cedex A (France)

Tél. + 33 (0)169181514 - Fax + 33 (0)1 69860678

e-mail : abonnements@edpsciences.org

$\square$ Paiement en ligne disponible sur notre site www.aos-journal.org

$\square$ Paiement par carte bancaire : $\square$ Carte Visa $\square$ Master Card

$\square$ American Express

$\mathrm{N}^{\circ}$

Noter ici les 3 derniers chiffres situés au dos de la carte

Date d'expiration

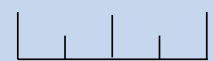

Signature obligatoire

\section{VOTRE ADRESSE :}

Vous êtes: $\square$ Chirurgien-Dentiste $\square$ Stomatologue $\square$ Orthodontiste $\square$ Autre: précisez

Nom :

Prénom :

Adresse :

Code Postal

Ville

Tél

Fax

E-mail 


\section{Association d'Enseignement d'Odontologie et de Stomatologie}

\section{LA VIE DE L'ASSOCIATION}

PRÉSIDENTS D'HONNEUR :

Pierre CERNÉA ${ }^{\dagger}$, Jean OUVRARD ${ }^{\dagger}$, Michel BENOIST ${ }^{\dagger}$,

Hubert OUVRARD

MEMBRES D'HONNEUR :

A. RICHARD ${ }^{\dagger}$, H. LENTULO ${ }^{\dagger}$,

A. LAMBERT, P. GONON ${ }^{+}$,

J.-P. RAGOT ${ }^{\dagger}$

Ch. DESCROZAILLES ${ }^{\dagger}$,

G. FICHELLE

\section{CONSEIL D'ADMINISTRATION}

PRÉSIDENT : LUC CHIKHANI

VICE-PRÉSIDENT :

Jean-François LEGRAND

SECRÉTAIRE GÉNÉRAL :

Frédéric LARCHÉ

TRÉSORIER :

Gérard PASQUET

MEMBRES :

Jacques-Charles BERTRAND, Danielle BUCH, Jean BUQUET, Estelle FAVRE, Jean MEYER, Guy PRINC, Valérie TRAVERT

\begin{abstract}
COMITÉ SCIENTIFIQUE
PRÉSIDENT : LUC CHIKHANI

VICE-PRÉSIDENT : Hubert OUVRARD

SECRÉTAIRE : Frédéric LARCHÉ

MEMBRES : Christophe BONNEFOY, Estelle FAVRE, Gilles FLEURIDAS, Thierry GUÉRIN, Jean-François LEGRAND, Frank LEVAVASSEUR, Hervé MISSISTRANO, Gérard PASQUET, Thierry PIRAL, Valérie TRAVERT
\end{abstract}

ANCIENS PRÉSIDENTS : P. CERNÉA ${ }^{\dagger}$, P. HENNION, H. LENTULO ${ }^{\dagger}$, M. LEPOIVRE ${ }^{\dagger}$, A. MARMASSE ${ }^{\dagger}$, $\begin{array}{lll}\text { A. LAMBERT, R.-R. RIGOLET } & \\ & \text { A. R. BATAILLE } \\ & \end{array}$ L.-A. STIEGLER ${ }^{\dagger}$, F. BOUCHON ${ }^{\dagger}$, Ch. DESCROZAILLES, P. FRIEZ ${ }^{\dagger}$, F. BROCHERE ${ }^{\dagger}$, A. RICHARD ${ }^{\dagger}$, R.-L. NINET', M. CHATEAU, P. COUSTAING, A. MUGNIER, R. WEILL ${ }^{\dagger}$

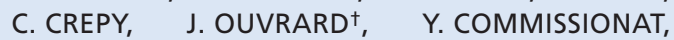
B. DANGY ${ }^{\dagger}$, F. GARLOPEAU ${ }^{\dagger}$, J.-L. DEPHILIPPE, J. VIGNEUL, H. PETIT, M. BENOIST ${ }^{\dagger}$, G. FICHELLE, J. LAUFER, J.-P. SANTORO, P. LAUDENBACH ${ }^{\dagger}$, P. DARGENT ${ }^{\dagger}$, CI. SCHUHMANN, F. MAESTRONI ${ }^{\dagger}$, J.-P. DEFFEZ，H. OUVRARD， D. RIGOLET，J. BUQUET, J.-P. RAGOT
 , R. BUGUGNANI, P. BORDAIS, J.-M. LAURICHESSE', J.-Ch. BERTRAND, J.-F. LEGRAND, G. PASQUET, J. DICHAMP, D. BUCH， A. DEBOISE', C. BOZON, F. LARCHÉ, M. AMORIC, G. PRINC, V. TRAVERT, J.-P. LÉZY, F. LEVAVASSEUR, E. FAVRE, H. MISSISTRANO, T. PIRAL, C. BONNEFOY

\section{BULLETIN D'ADHÉSION Á L'A.E.O.S.}

$\square$ Oul, je souhaite devenir membre de l'A.E.O.S. Ci-joint le règlement de ma cotisation annuelle $2010: 20 €$ au lieu de $60 €$ (offre réservée aux nouveaux abonnés des A.O.S.).

Je règle par chèque à l'ordre de l'A.E.O.S.

$\mathrm{Dr}$ :

Prénom :

Adresse

Code postal :

Ville :

Téléphone :

Fax :

Bulletin à retourner à : I'Association d'Enseignement d'Odontologie et de Stomatologie (A.E.O.S.) 179, rue Saint-Honoré - 75001 Paris - Tél. 0142605032 - Fax 0147034643 